\title{
\begin{tabular}{|l|} 
EXPERT \\
REVIEWS \\
\hline
\end{tabular}
}

\section{Assessment and detection of pain in noncommunicative severely brain-injured patients}

Expert Rev. Neurother. 10(11), 1725-1731 (2010)

\section{Caroline Schnakers ${ }^{\dagger 1}$ Camille Chatelle', Steve Majerus², Olivia Gosseries", Marie De $\mathrm{Val}^{3}$ and Steven Laureys ${ }^{1,4}$}

${ }^{1}$ Coma Science Group, Cyclotron Research Centre, Sart Tilman, B30, University of Liège, 4000 Liège, Belgium

${ }^{2}$ Department of Cognitive Sciences, University of Liège, Liège, Belgium ${ }^{3}$ Department of Intensive Care, University Hospital of Charleroi, Charleroi, Belgium

${ }^{4}$ Department of Neurology, University Hospital of Sart Tilman, Liège, Belgium ${ }^{+}$Author for correspondence:

Tel.: +3243662303

Fax: +3243662946 c.schnakers@ulg.ac.be

Detecting pain in severely brain-injured patients recovering from coma represents a real challenge. Patients with disorders of consciousness are unable to consistently or reliably communicate their feelings and potential perception of pain. However, recent studies suggest that patients in a minimally conscious state can experience pain to some extent. Pain monitoring in these patients is hence of medical and ethical importance. In this article, we will focus on the possible use of behavioral scales for the assessment and detection of pain in noncommunicative patients.

KeYwords: behavioral scale $\bullet$ coma $\bullet$ consciousness $\bullet$ minimally conscious state $\bullet$ nociception $\bullet$ pain $\bullet$ severe brain injury $\bullet$ vegetative state

Detecting pain in severely brain-injured patients with disorders of consciousness represents a real challenge [1]. Pain assessment is usually based on the patient's verbal report, as pain is a subjective first-person experience [2]. However, patients recovering from coma cannot express their feelings and potential experience of pain. More exactly, while vegetative patients only show reflexive activity, patients in a minimally conscious state demonstrate inconsistent, elementary but reproducible signs of consciousness, and can sometimes verbalize, but they do not show functional communication that could be used for pain assessment [3]. Recent studies suggest that minimally conscious patients can experience pain to some extent [4]. It is hence of medical and ethical importance to assess and detect pain in these patients. In a previous article, we have presented remnant neural correlates, treatment and ethical aspects linked to pain in patients recovering from coma [1]. At that time, no tool existed to specifically assess pain in these patients. Nevertheless, recently, a behavioral scale, the Nociception Coma Scale, has been developed to detect pain in patients with disorders of consciousness [5]. With regard to this recent publication, in this article we will also focus on the possible use of behavioral scales for the assessment and detection of pain in noncommunicative patients.

\section{Definitions of vegetative \& minimally conscious states}

The term 'vegetative' suggests a preservation of autonomic functions (e.g., cardiovascular, respiratory and thermoregulation functions) and re-emergence of the sleep-wake cycle (i.e., periods of spontaneous eyes opening) [6]. The vegetative state (VS) often results from bihemispheric injury involving the white matter, or from bilateral lesions in the thalamus with sparing of the brainstem, hypothalamus and basal ganglia. Behaviorally, there is no evidence of awareness of self or environment, no response to sensory stimuli suggesting volition or conscious purpose and no evidence of language comprehension or meaningful expression. Infrequently, behaviors such as inappropriate smiling, crying or grimacing can be reported in patients diagnosed with VS [7]. With careful assessment, it is possible to demonstrate that these behaviors are not voluntary or goal directed. Establishing a definitive prognosis is difficult; however, when this state lasts 1 month or more, the patient is considered in a 'persistent' VS. When it lasts more than 3 months (for nontraumatic etiologies) or 1 year (for traumatic etiology), the patient can be considered in a 'permanent' VS [6].

On the contrary, the minimally conscious state (MCS) is characterized by the presence of inconsistent, but clearly discernible, behavioral signs of consciousness [3]. Such signs must be reproducible within a given examination, although behavior may fluctuate across examinations. Diagnostic criteria include: inconsistent response to verbal order; localization to noxious stimuli; automatic movements (e.g., scratching); environmentally contingent emotional responses; 
object localization and manipulation; sustained visual fixation and pursuit; verbalizations; and intentional but unreliable communication. Regarding prognosis, the probability of functional recovery at 1 year following traumatic brain injury is significantly more favorable for MCS patients relative to VS patients ( 50 vs $3 \%$ attaining moderate disability) [8]. Some patients in MCS progress slowly, while others remain in this condition permanently. Unlike VS, clearly defined temporal parameters for recovery do not exist, and there is a wide heterogeneity in the degree of functional recovery ultimately attained $[9,10]$. Emergence from MCS occurs when the patient is able to reliably communicate through verbal or gestural yes-no responses, or is able to demonstrate the use of two or more objects in a functional manner [3].

\section{Pain processing in vegetative versus minimally conscious patients}

Pain is mediated by a lateral and medial widely distributed cerebral network. A distinction must be made between brain areas involved in pain perception per se versus suffering as related to the conscious perception of the pain in question. Activation of the lateral pain system, including lateral thalamus, primary and secondary somatosensory cortex, parietal operculum and insula, are related to the sensory-discriminative aspects of pain processing. With regards to the medial pain system, the descending connections of the anterior cingulate cortex to medial thalamic nuclei and to periaqueductal matter are thought to be involved in the modulation of reflex responses to noxious stimuli, whereas cingulate, amygdala, hippocampus, hypothalamus, locus coeruleus but also orbitofrontal and medial prefrontal cortices are thought to be involved with pain-related affective behavior. Finally, interconnectivity between the periaqueductal matter and orbitofrontal cortex may be key to cognitive-emotional responses associated with pain. Thus, the central pain control processes seem to concern the cognitive-evaluative, motivational-affective and sensory-discriminative systems that characterize the pain response [11,12].

At present, the vast majority of neuroscientific evidence points to the critical role of thalamo-cortical interactions subserving conscious experience [13]. The question of whether consciousness is required for sensory perception, including pain perception and suffering in persons with disorders of consciousness, has certainly been posited, particularly related to the VS. Laureys et al. investigated central processing of pain stimuli by using PET imaging [14]. Electrical stimulations of the median nerve were administered to $15 \mathrm{VS}$ patients and changes in regional cerebral blood flow were compared with 15 healthy controls. Noxious stimulation activated midbrain, contralateral thalamus and primary somatosensory cortex in all VS patients.
Moreover, the activated primary somatosensory cortex was functionally disconnected from secondary somatosensory, bilateral posterior parietal, premotor, polysensory superior temporal and prefrontal cortices. Primary cortex activation appeared to be isolated from activity in the higher-order associative cortex. These results were replicated by Kassubeck et al. with seven anoxic patients in a VS [15]. Hypermetabolism was found in the posterior insula/secondary somatosensory cortex, postcentral gyrus/primary somatosensory cortex and the cingulate cortex contralateral to the stimulus and in the posterior insula ipsilateral to the stimulus. The functional disconnections in cortico-thalamo-cortical (between thalamus and frontal cortex) pathways suggest that cortical processes in the VS may occur as a primary, isolated and disconnected processing phenomena, reducing the likelihood that painful stimuli are experienced in an integrated and conscious manner [16]. The cerebral activation to pain is different in MCS patients. Boly et al. showed brain activation similar to controls in response to noxious stimuli in five MCS patients [4]. The activation of the anterior cingulate area suggests that these patients may perceive the unpleasant aspects of painful stimulation, since the anterior cingulate is thought to support affective appraisal of pain stimulation. Intact connectivity between primary and associative cortices has also been observed in these patients, suggesting the existence of integrated and distributed neural processing, and possibly the existence of conscious pain perception in MCS patients (FIGURE 1).

\section{Pain assessment in noncommunicative patients}

Even if they present some level of consciousness, MCS patients are unable to consistently or reliably communicate their feelings and possible conscious pain perception. Hence, it is of utmost

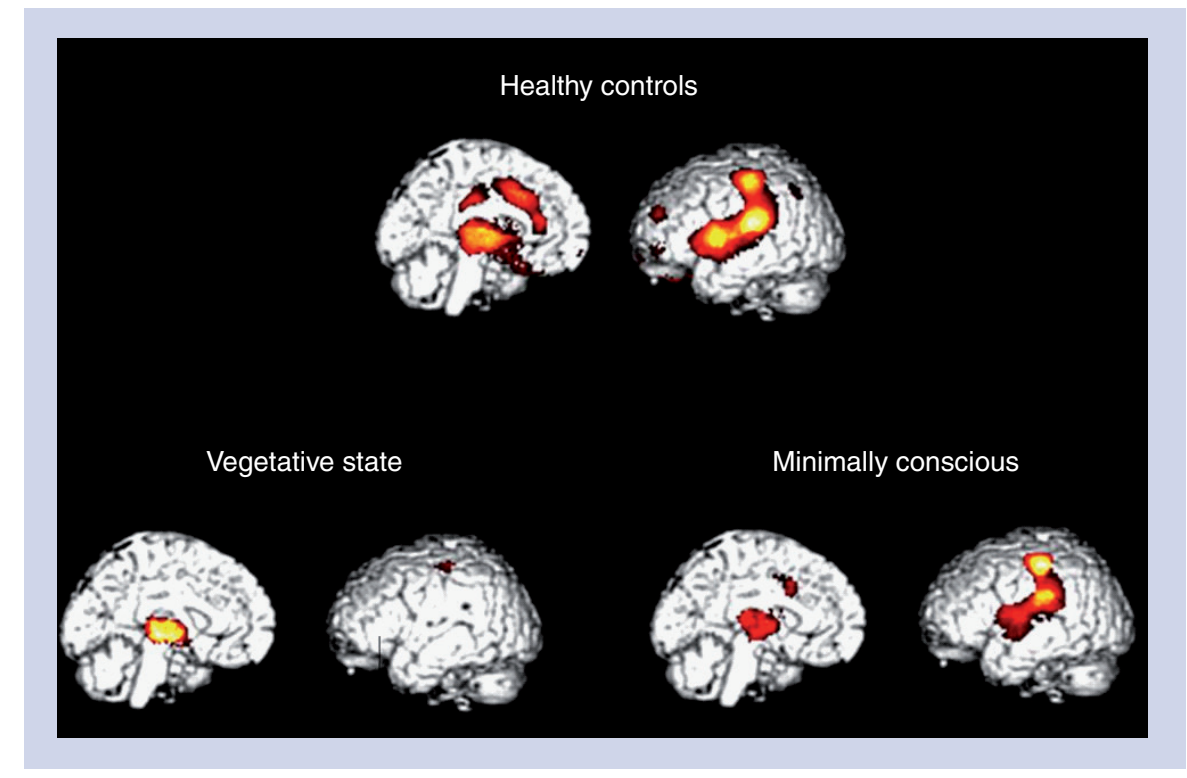

Figure 1. Cerebral activation to noxious stimulation. In red, brain regions that activated more during noxious stimulation in healthy controls, in vegetative state and in minimally conscious state as compared with at rest.

Adapted from $[4,14]$. 
importance to develop sensitive tools to assess the level of pain perception in these patients. When assessing awareness, various language, auditory, visual and somatosensory, as well as noxious, stimuli are administered and the patients' responses to these stimulations are rated as a function of voluntary controlled responses versus reflex activity. During these assessments, three types of motor responses are distinguished following the application of a noxious stimulus: stereotypical responses (i.e., slow stereotyped flexion or extension of the upper and lower extremities), flexion withdrawal and localization responses. These responses are linked to brainstem, subcortical and cortical activity, respectively [17]. Typical assessment procedures of consciousness level in these patients do not consider other pain-related behaviors. For another population of noncommunicative patients, such as the demented elderly, newborns/preverbal children or sedated/intubated patients, more specific pain scales have been developed and validated.

For the demented elderly, Zwakhalen and coworkers have recently identified 12 observational pain assessment scales. Most of these scales were under development and show moderate psychometric qualities. Nevertheless, four scales presented higher psychometric qualities: the DOLOPLUS2; the Pain Assessment Checklist for Seniors with Limited Ability to Communicate (PACSLAC); the Echelle Comportementale pour Personnes Agées (ECPA); and the Pain Assessment in Advanced Dementia scale (PAINAD) [18]. The DOLOPLUS2 is one of the scales most used in old noncommunicative patients. It assesses ten items according to three aspects (i.e., somatic, psychomotor and psychosocial aspects). Pain is considered as present with a score of 5, a maximum score of 30 reflecting an intense painful experience [19]. The DOLOPLUS2 is usually not used to represent pain at a specific moment, but is used to reflect the progression of pain. Therefore, this scale is interesting in long-term care where a patient can be followed for a sufficient amount of time. The PACSLAC is a list of 60 behaviors that are divided into different subgroups: facial expression; movements of the body; and physiological indicators, such as changes in sleep or appetite. Each item is scored in a dichotomous way (i.e., present or absent) with a total score ranging from 0 to 60 [20]. As with the DOLOPLUS2, this scale has to be used in long-term care. Its administration nevertheless needs more time, and the total score does not refer to any level of pain. Therefore, the PACSLAC seems less interesting at a clinical level. The ECPA is a behavioral scale for communicative and noncommunicative elderly. The version for noncommunicative patients consists of 11 items divided into two periods of observation: before care and during care. The total score ranges from 0 (absence of pain) to 44 (intense pain) [21]. By comparing the intensity of pain before and during care, the ECPA allows the assessment of the intensity of pain without long-term followup. In contrast to the DOLOPLUS2 and PACSLAC, this scale can, therefore, also be used in short-term care. The PAINAD was developed to assess pain in patients with severe dementia. It includes five items (i.e., breathing, negative vocalizations, facial expression, body movements and consolability) scored between 0 and 2, a score of 0 reflecting an absence of pain [22]. As with the ECPA, this scale can be used without knowing the patient's usual responses. The PAINAD is also easier to administer as it is simple to understand and can be used after a short training.

For newborns and preverbal children, Hummel and coworkers have identified 16 scales that are usually based on behavioral (i.e., facial action, body movement and tone, cry, state/sleep and consolability), as well as physiological, indicators of pain (i.e., increased heart rate, respiratory rate, blood pressure, decreased heart rate variability and oxygen desaturation) [23]. According to Herr and coworkers, none of these behavioral scales have been shown to possess better psychometric qualities than others. Clinicians should select a scale that is appropriate to the patient and types of pain (e.g., procedural or postoperative) [24]. Among these scales, we should nevertheless highlight some of the most known ones. One is the Premature Infant Pain Profile (PIPP), which was validated to measure pain during invasive care in premature newborns [25]. This scale takes into account the newborn's age and compares behaviors before and after painful stimulation. It scores from 0 to 21 ; a score of 12 suggesting that pain management is necessary. Its use is nevertheless complex, which limits its clinical interest. The Neonatal Infant Pain Scale (NIPS) is a useful tool to observe premature newborns during painful procedures [26]. Its scores vary between 0 (absence of pain) and 7 (severe pain); a score of 3 reflecting the presence of a painful experience. In contrast to the PIPP, this scale is easy to use. Finally, the Faces, Legs, Activity, Cry, Consolability (FLACC) pain assessment tool can be used in preverbal children aged between 2 months and 7 years who cannot report pain [27]. This scale is recommended to assess postsurgical pain. As with the NIPS, this scale is easy to use. This scale does not have defined pain thresholds; however, its score ranging from 0 to 10 may facilitate its interpretation.

For sedated/intubated patients, Pudas-Tahka and coworkers recently identified five pain assessment scales that included behavioral and physiological indicators. However, their psychometric properties varied, and it was not possible to deduce their clinical utility [28]. We will nevertheless cite several scales, such as the COMFORT scale, the Behavioral Pain Scale (BPS) and a new tool called the DOLOUSI. The COMFORT scale can be used in young sedated patients between 0 and 3 years old [29]. It includes the observation of respiratory and motor responses, cardiac frequency, blood pressure, facial expression, agitation and level of awakening. Each parameter is scored from 1 to 5 . The total score is ranged from 8 to 40 ; a score between 17 and 26 indicating an appropriate sedation. This is the sole scale that assesses oversedation, comfort and distress in newborns and young children in intensive care. The BPS assesses facial expression, movements of the upper limbs and the compliance to mechanical ventilation in intubated adults [30]. Each parameter is scored from 1 to 4. The total score ranges from 4 to 12 . Until now, the BPS represents the sole validated scale for adults. Nevertheless, recently, the DOLOUSI pain scale has been developed in order to assess pain in noncommunicative critically ill patients sedated/intubated and hospitalized in intensive care units. This scale is a behavioral, unidimensional scale based upon the assessment of four items: adaptation to mechanical ventilation, facial expression, motricity 
and tears. The total scores range from 4 to 15 ; a score of 7 suggesting significant level of pain and a need for appropriate pain management [31]. Several psychometric parameters have been assessed with the DOLOUSI. Indeed, De Val et al. recently conducted a preliminary study in 110 noncommunicative sedated/intubated patients. In total, 196 assessments were collected at rest, during care with low (i.e., eye care) and high (i.e., complete care with lateral decubitus) pain. A significantly higher score was obtained for the care with high pain, in contrast to the Ramsay Sedation Scale, which is often used in intensive care for monitoring patients' sedation level [32]. Finally, an excellent inter-rater agreement, as well as an excellent internal consistency, was observed [33]. Therefore, the DOLOUSI seems to be a promising tool to assess pain in sedated/ intubated patients hospitalized in intensive care units. Further investigations are nevertheless needed in order to compare this scale to an existing scale, such as the BPS.

Even if pain scales have been developed for different types of noncommunicative populations, none of these are adapted to detect pain in patients recovering from coma. In this context, the Nociception Coma Scale (NCS) has recently been developed to assess pain in patients recovering from coma [5]. This scale consists of the observation of motor, verbal and visual responses to pain stimulation, as well as facial expression. Its total score ranges from 0 to 12 (Box 1). Initially, breathing responses were also assessed, but later discarded due to the difficulty to reliably assess breathing patterns in patients not benefiting from respiratory monitoring devices [31]. Previous studies have also demonstrated that autonomic changes, such as respiration and heart rate, are not reliable indicators of pain $[34,35]$. The validation study of the NCS was a prospective multicentric study with patients recruited from acute care, neurology, neurorehabilitation and nursing home centers. It

\section{Box 1. Protocol of the Nociception Coma Scale.}

\section{Motor response}

- 3 - Localization to noxious stimulation

- 2 - Flexion withdrawal

- 1 - Abnormal posturing

- 0 - None/flaccid

\section{Verbal response}

- 3 - Verbalization (intelligible)

- 2 - Vocalization

- 1 - Groaning

- 0 - None

Visual response

- 3 - Fixation

- 2 - Eyes movements

- 1 - Startle

- 0 - None

Facial expression

- 3-Cry

- 2 - Grimace

- 1 - Oral reflexive movement/startle response

- 0 - None was performed by observing the responses of 48 severely braininjured patients (28 VS and 20 MCS; age range 20-82 years; 17 of traumatic etiology) to a noxious stimulation (i.e., pressure applied to the fingernail). The results demonstrated a good interrater agreement and a good correlation between the NCS and other validated pain scales, such as, for instance, the NIPS, the FLACC and the PAINAD, suggesting that, in parallel to these scales, the NCS assesses pain. However, on the contrary to these pain scales, the NCS scores were significantly different according to clinical entity (i.e., VS and MCS), suggesting that the NCS scale is particularly suited for the assessment of pain in patients recovering from coma. Chatelle et al. explored the specificity of the NCS in 25 postcomatose patients (11 VS and 14 MCS; age range 15-82 years; ten of traumatic etiology) [36]. They compared the NCS total scores at rest, in response to noxious and non-noxious tactile (i.e., tap on the shoulder) stimulations. Significant differences between total scores obtained at rest and following nociceptive stimulations, but also between tactile and nociceptive conditions, were mainly found. No difference was found between baseline and tactile conditions (Figure 2). These results demonstrate that the NCS is a sensitive scale that specifically assesses nociception in severely brain-injured patients. In a case study, we recently assessed the sensitivity of the scale to the effects of antalgic treatment in a 36-year-old MCS patient following traumatic brain injury. This patient presented a severe spleen lesion and had a score of 6 at the NCS during care (i.e., flexion withdrawal, oral reflexive movement and visual fixation). Antalgic treatment (i.e., $50 \mathrm{mg}$ of Dolzam ${ }^{\circledR}$ [tramadol] and $50 \mathrm{mg}$ of Litican ${ }^{\circledR}$ [alizapride hydrochloride]) was administered, and the NCS performed $1 \mathrm{~h}$ later showed a score of 0 . Even if further clinical validation is needed, the NCS seems to be a promising tool for assessing and monitoring pain in severely brain-injured patients and seems to be adapted not to all noncommunicative populations, but specifically to VS and MCS patients.

\section{Expert commentary}

Minimally conscious state patients cannot, by definition, consistently or reliably communicate their feelings and possible conscious pain perception. They nevertheless present, to some extent, preserved and integrated brain processing in response to noxious stimulation, suggesting possible conscious experience of pain. The existence of tools for detecting pain in these patients is, therefore, of medical and ethical importance. Behavioral scales such as the NCS have been developed for assessing pain in severely brain-injured patients, and will allow a better specification of the behavioral patterns linked to pain experience in MCS and VS patients (e.g., prevalence of grimaces in VS versus MCS patients) and to the monitoring of pain management in order to avoid over- or under-medication. Finally, in the future, more attention should be paid to the ethical implications of pain detection and treatment in disorders of consciousness.

\section{Five-year view}

First, future research will need to address the relationship between pain perception and the experience of suffering in patients in a MCS. Pain perception must be differentiated from suffering, as 


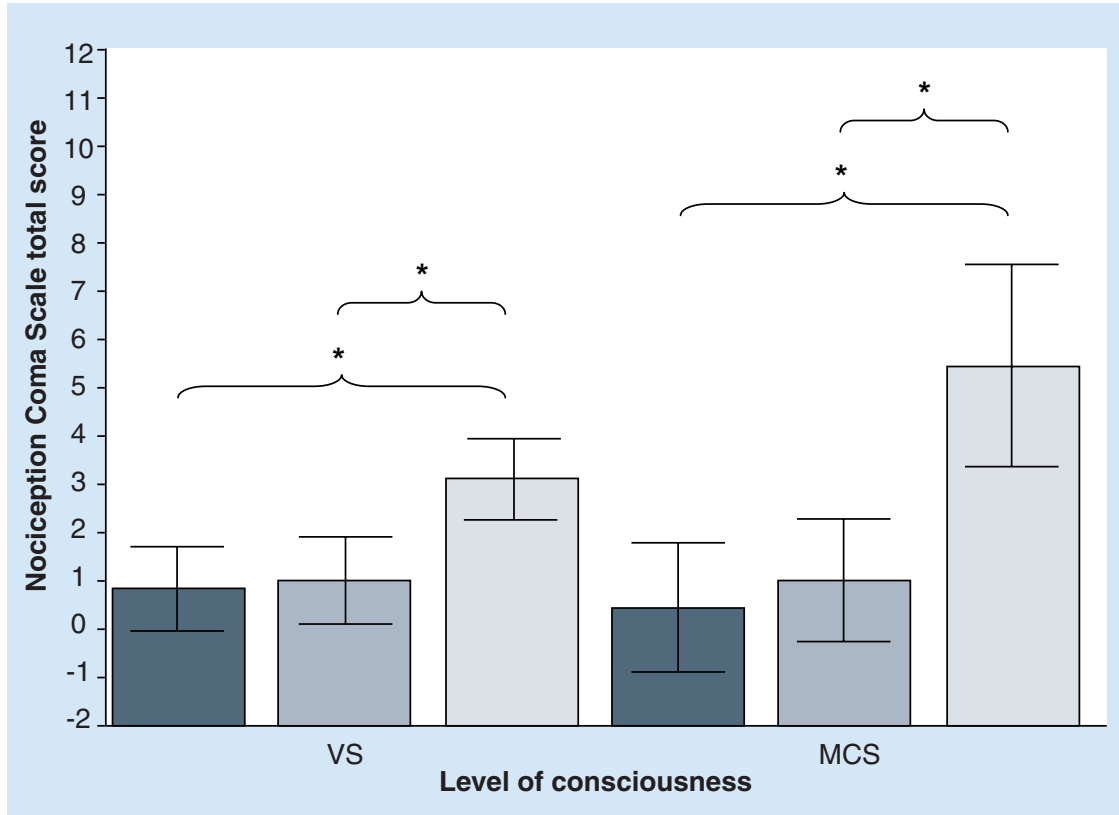

Figure 2. Mean (and standard deviation) of Nociception Coma Scale total scores (score range: $0-12$ ) at rest (dark gray), following tactile stimulation (mid-gray) and following noxious stimulation (light gray) in vegetative and minimally conscious patients. Asterisk marks significant difference between condition $(p<0.01)$. MCS: Minimally conscious state; VS: Vegetative state. in order to better characterize the cognitive pattern existing in MCS and, therefore, the potential suffering experienced by these patients. Additional research is also warranted for VS patients. The question of the grimaces as an indicator of pain should also be addressed. Indeed, even if grimacing is considered as an indicator of pain in scales employed in the demented elderly and newborns, the Multi Society Task Force [6] does not consider this behavior as a necessary sign of conscious perception. Patients showing no sign of consciousness except grimaces to stimuli can, therefore, be diagnosed as being in a VS according to established diagnostic criteria. No functional neuroimaging study has yet investigated the neural processing of pain in these patients, as previous studies did not involve VS patients presenting grimaces in response to pain. Additional research is needed to better understand the neural correlates underlying this potential indicator of painful experience.

Further investigation is also needed to raise the clinician's awareness of the the latter involves a complex cognitive-affective phenomenon, involving not only a negative emotional response to the pain experience, but also the ability to remember that particular experience or set of experiences. Schnakers et al. showed that MCS patients are able to show complex remnant cognitive functioning using an active evoked-related potentials paradigm [37]. A larger P3 response was observed in the active condition (i.e., where the patient was asked to count a target) compared with the passive condition (i.e., where the patient was just hearing this target), suggesting that some high-level cognitive treatment could be preserved in patients recovering from coma, even in the presence of a low behavioral pattern (some only showed oriented eye movements). Other studies nevertheless need to be performed existence of scales, such as the NCS, to be used during pain management of these challenging noncommunicative patient groups. Clinical guidelines concerning pain do not currently exist. A patient named Terri Schiavo died from dehydration without administration of opiates as she was diagnosed as VS by the High Court's experts [38]. According to a European survey conducted on 2059 medical and paramedical professionals on possible pain perception in patients recovering from coma, half of the respondents think that patients in a VS may feel pain $(56 \%$ of the medical doctors and $68 \%$ of the paramedical caregivers) [39]. Analgesic treatment might nevertheless show some side effects. A systematic use of an analgesic could have undesirable effects (sedation) and, therefore, result in the

\section{Key issues}

- Detecting pain in noncommunicative patients recovering from coma represents a real challenge, as pain assessment is usually based on the patient's verbal report.

- Whereas vegetative state (VS) patients only show reflexive activity, patients in a minimally conscious state (MCS) demonstrate inconsistent, elementary but reproducible signs of consciousness, and can sometimes verbalize, but they do not show functional communication that could be used for pain assessment.

- Intact connectivity between primary and associative cortices also suggests the existence of an integrated and distributed neural processing, and possibly the existence of conscious pain perception in MCS patients.

- Specific pain scales have been developed and validated for noncommunicative patients, such as the demented elderly, newborns/preverbal children or sedated/intubated patients.

- The Nociception Coma Scale has recently been developed to assess pain in patients recovering from coma. The results demonstrated a good inter-rater agreement, concurrent validity and sensitivity.

- Behavioral scales such as the Nociception Coma Scale will allow, in the future: a better specification of the behavioral patterns linked to pain experience in MCS and VS patients (e.g., prevalence of grimaces in VS versus MCS patients); and the monitoring of pain management in order to avoid over- or under-medication. 
underestimation of the level of consciousness of these patients. Adequately assessing and monitoring pain represents a true challenge. Evidence-based guidelines for the management of possible pain perception and suffering in patients recovering from coma are strongly needed.

The use of a behavioral scale is crucial to assess and detect pain. However, additional paramedical tools need to be developed in order to help clinicians in improving its detection. Recently, Haenggi and coworkers evaluated electroencephalographic (EEG) parameters as an adjunct to monitoring the effects of commonly used sedative and analgesic drugs and intratracheal suctioning in critically ill patients [40]. According to their results, painful stimuli and sedative and analgesic drugs are associated with significant changes in EEG parameters, suggesting that it could be integrated to pain management. Previous studies have demonstrated that EEG parameters could help to some extent in determining the level of consciousness in severely brain-injured patients [41,42]. However, no study has investigated its efficacy in detecting pain. Further investigations are hence needed. Other paramedical tools aiming to improve communication in these patients could also help in detecting pain. Owen et al. recently described the use of functional MRI to detect consciousness in a patient diagnosed as being in a VS [43]. The patient was instructed to imagine spatial navigation and motor imagery tasks. No differences were found in terms of brain activation between the VS patient and the healthy volunteers. This technique may permit the identification of intentional brain activation at the single subject level, without requiring a reliable motor response. It may also allow a form of communication with these patients and might serve as a better surrogate for the detection of pain [44].

\section{Financial \& competing interests disclosure}

The authors' research is funded by the Belgian Fund for Scientific Research (FRS-FNRS), European Commission, James McDonnell Foundation, Mind Science Foundation, French Speaking Community Concerted Research Action (ARC-06/11-340), Fondation Médicale Reine Elisabeth, University of Liège and the CNRS/FNRS-CGRI collaboration funds. The authors have no other relevant affiliations or financial involvement with any organization or entity with a financial interest in or financial conflict with the subject matter or materials discussed in the manuscript apart from those disclosed.

No writing assistance was utilized in the production of this manuscript.

\section{References}

Papers of special note have been highlighted as:

- of interest

•• of considerable interest

1 Schnakers C, Zasler ND. Pain assessment and management in disorders of consciousness. Curr Opin Neurol 20(6), 620-626 (2007).

- Examines the current knowledge (remnant neural correlates of pain and pain treatment) for guiding decisions regarding management of pain in persons with disorders of consciousness.

2 IASP. Classification of Chronic Pain: Descriptions of Chronic Pain Syndromes and Definitions of Pain Terms. Task Force on Taxonomy. IASP Press, WA, USA (1994).

- Presents chronic pain diagnosis, descriptions of pain syndromes and detailed pain definitions.

-3 Giacino J, Ashwal S, Childs N et al. The minimally conscious state: definition and diagnostic criteria. Neurology 58(3), 349-353 (2002).

- Presents diagnostic criteria for the minimally conscious state (MCS).

- 4 Boly M, Faymonville ME, Schnakers C et al. Perception of pain in the minimally conscious state with PET activation: an observational study. Lancet Neurol. 7(11), 1013-1020 (2008).
- In this study, cerebral correlates of pain processing are found in a similar network in controls and patients in MCS but are much more widespread than those of patients in persistent vegetative state.

5 Schnakers C, Chatelle C, Vanhaudenhuyse A et al. The Nociception Coma Scale: a new tool to assess nociception in disorders of consciousness. Pain 148(2), 215-219 (2010).

- Proposes a new scale developed for assessing nociception in coma survivors, the Nociception Coma Scale, and explores its concurrent validity, inter-rater agreement and sensitivity.

6 The Multi-Society Task Force on PVS. Medical aspects of the persistent vegetative state (1). N. Engl. J. Med. 330 (21), 1499-1508 (1994).

- Presents diagnostic criteria for the vegetative state.

7 Working Party of the Royal College of Physicians. The vegetative state: guidance on diagnosis and management. Clin. Med. 3(3), 249-254 (2003).

8 Giacino J. The vegetative and minimally conscious states: a comparison of clinical features and functional outcome. J. Head Trauma Rehabil. 12, 36-51 (1997).

-9 Fins JJ, Schiff ND, Foley KM. Late recovery from the minimally conscious state: ethical and policy implications. Neurology 68(4), 304-307 (2007).
10 Lammi MH, Smith VH, Tate RL et al. The minimally conscious state and recovery potential: a follow-up study 2 to 5 years after traumatic brain injury. Arch. Phys. Med. Rehabil. 86(4), 746-754 (2005).

- 11 Borsook D, Becerra LR. Breaking down the barriers: $\mathrm{fMRI}$ applications in pain, analgesia and analgesics. Mol Pain 2, 30 (2006).

- Summarizes functional MRI findings that have led to our current knowledge on acute and chronic pain, and also treatment.

12 May A. Neuroimaging: visualising the brain in pain. Neurol. Sci. 28 (2), 101-107 (2007).

- The complex network of cortical and subcortical brain structures underlying the pain matrix is reviewed regarding the sensory-discriminative dimensions of pain.

13 Baars B, Ramsoy T, Laureys S. Brain, conscious experience and the observing self. Trends Neurosci. 26, 671-675 (2003).

14 Laureys S, Faymonville ME, Peigneux P et al. Cortical processing of noxious somatosensory stimuli in the persistent vegetative state. Neuroimage 17(2), 732-741 (2002).

-. In this study, noxious stimulation of persistent vegetative state patients resulted in increased neuronal activity in the primary somatosensory cortex, which seems to be isolated and dissociated from higher-order associative cortices. 
15 Kassubek J, Juengling FD, Els T et al. Activation of a residual cortical network during painful stimulation in long-term postanoxic vegetative state: a $15 \mathrm{O}-\mathrm{H} 2 \mathrm{O}$ PET study. J. Neurol. Sci. 212(1-2), 85-91 (2003).

16 Laureys S, Goldman S, Phillips C et al. Impaired effective cortical connectivity in vegetative state: preliminary investigation using PET. Neuroimage 9(4), 377-382 (1999).

17 Stevens RD, Nyquist PA. Coma, delirium, and cognitive dysfunction in critical illness. Crit. Care Clin. 22(4), 787-804 (2006).

- 18 Zwakhalen SM, Hamers JP, Abu-Saad HH et al. Pain in elderly people with severe dementia: a systematic review of behavioural pain assessment tools. $B M C$ Geriatr. 6, 3 (2006).

- Systematic review that identifies pain assessment scales for elderly people with severe dementia and evaluates the psychometric properties and clinical utility of these instruments.

19 Lefebre-Chapiro L. The Doloplus 2 scale-evaluating pain in the elderly. Eur. J. Pall. Care 8, 191-194 (2001).

-20 Fuchs-Lacelle S, Hadjistavropoulos T. Development and preliminary validation of the pain assessment checklist for seniors with limited ability to communicate (PACSLAC). Pain Manag. Nurs 1, 37-49 (2004).

-21 Morello R, Jean A, Alix M, Sellin-Peres D, Fermanian J. A scale to measure pain in non-verbally communicating older patients: the EPCA-2 Study of its psychometric properties. Pain 133(1-3), 87-98 (2007).

-22 Warden V, Hurley AC, Volicer L. Development and psychometric evaluation of the Pain Assessment in Advanced Dementia (PAINAD) scale. J. Am. Med. Dir. Assoc. 4(1), 9-15 (2003).

-23 Hummel P, Van Dijk M. Pain assessment: current status and challenges. Semin. Fetal Neonatal Med. 11(4), 237-245 (2006).

-24 Herr K, Coyne PJ, Key T et al. Pain assessment in the nonverbal patient: position statement with clinical practice recommendations. Pain Manag. Nurs. 7 , 44-52 (2006).
- Presents the position statement and clinical practice recommendations for pain assessment in noncommunicative patients.

-25 Stevens B, Johnston C, Petryshen P, Taddio A. Premature infant pain profile : development and initial validation. Clin. J. Pain 12, 13-22 (1996).

-26 Lawrence J, Alcock D, McGrath P et al. The development of a tool to assess neonatal pain. Neonatal Netw. 12(6), 59-66 (1993).

27 Merkel SI, Voepel-Lewis T, Shayevitz JR et al. The FLACC: a behavioral scale for scoring postoperative pain in young children. Pediatr. Nurs. 23(3), 293-297 (1997).

-28 Pudas-Tahka SM, Axelin A, Aantaa R et al. Pain assessment tools for unconscious or sedated intensive care patients: a systematic review. J. Adv. Nurs. 65(5), 946-956 (2008).

- Report of a systematic review describing instruments developed for pain assessment in unconscious or sedated intensive care patients.

-29 Ambuel B, Hamlett KW, Marx CM. Assessing distress in pediatric intensive care environments : the Comfort Scale. J. Pediatr. Psychol. 17, 95-109 (1992).

- 30 Payen JF, Bru O, Bosson JL et al. Assessing pain in critically ill sedated patients by using a behavioral pain scale. Crit. Care Med. 29, 2258-2263 (2001).

- 31 Chatelle C, Vanhaudenhuyse A, Mergam AN et al. Pain assessment in noncommunicative patients. Rev. Med. Liege 63(5-6), 429-437 (2008).

32 Sessler CN, Grap MJ, Ramsay MA. Evaluating and monitoring analgesia and sedation in the intensive care unit. Crit. Care 12 (3), 2 (2008).

33 De Val M, Pachioli M, Lorent C et al. Assessment of pain in the intensive care unit in patients who cannot communicate: clinical interest of the SOS DOLOUSI, a Behavioural Pain Scale. Presented at: 3rd European Critical Care Nursing Congress, Florence, Italy, 9-11 October 2008.

34 Buttner W, Finke W. Analysis of behavioural and physiological parameters for the assessment of postoperative analgesic demand in newborns, infants and young children: a comprehensive report on seven consecutive studies. Paediatr. Anaesth. 10(3), 303-318 (2000).

- 35 Herr KA, Garand L. Assessment and measurement of pain in older adults. Clin. Geriatr. Med. 17(3), 457-478 (2001).

36 Chatelle C, Schnakers C,

Vanhaudenhuyse A et al. The Nociception Coma Scale to assess nociception in disorders of consciousness. Presented at: 8th World Congress on Brain Injury, Washington, USA, 10-14 March 2010.

- 37 Schnakers C, Perrin F, Schabus M et al. Voluntary brain processing in disorders of consciousness. Neurology 71(20), 1614-1620 (2008).

38 Wijdicks EF. Minimally conscious state vs. persistent vegetative state: the case of Terry (Wallis) vs the case of Terri (Schiavo). Mayo Clin. Proc. 81(9), 1155-1158 (2006).

- 39 Demertzi, A, Schnakers C, Ledoux D et al. Different beliefs about pain perception in the vegetative and minimally conscious states: a European survey of medical and paramedical professionals. Prog. Brain Res. 177, 329-338 (2009).

-• The authors provide results from a European survey on 2059 medical and paramedical professionals' beliefs on possible pain perception in patients with disorders of consciousness.

40 Haenggi M, Ypparila-Wolters H, Bieri C et al, Entropy and bispectral index for assessment of sedation, analgesia and the effects of unpleasant stimuli in critically ill patients: an observational study. Crit. Care 12(5), 186 (2008).

41 Schnakers C, Majerus S, Laureys S. Bispectral analysis of electroencephalogram signals during recovery from coma: preliminary findings. Neuropsychol. Rehabil. 15(3-4), 381-388 (2005).

42 Schnakers C, Ledoux D, Majerus S et al. Diagnostic and prognostic use of bispectral index in coma, vegetative state and related disorders. Brain Inj. 22 (12), 926-931 (2008).

43 Owen A, Coleman M, Boly M et al. Detecting awareness in the vegetative state. Science 313(5792), 1402 (2006).

44 Monti M, Vanhaudenhuyse A, Coleman MR et al. Willful modulation of brain activity in disorders of consciousness. N. Engl. J. Med. 362, 579-589 (2010). 